\title{
Test method of distribution functions of the semi-markovian system dwell times in states
}

\author{
Mikhail Zamoryonov ${ }^{1, *}$, Darya Zamoryonova ${ }^{1}$, and Vladimir Preis $^{2}$ \\ ${ }^{1}$ Sevastopol State University, Russian Federation, 299053, Sevastopol \\ ${ }^{2}$ Tula State University, Russian Federation, 300012, Tula
}

\begin{abstract}
The article presents and proves the theorem on the stay times in the trajectory states taking the repeated returns into account. A method for determining the distribution functions of the stay times of the semi-Markov system in the states, called the method of trajectories, is introduced. An example of semiMarkovian modeling systems proving the correctness of the proposed method is examined.
\end{abstract}

\section{Introduction}

In many cases the hierarchical approach is employed for modelling of the complex semi-Markovian (SM) systems for their model development. In this case it is necessary to abut separate elements of the system with each other, for what it is necessary to know the distribution functions (DF) of the operation times of these elements.

In the case of SM systems the integral equations of Markov renewal are solved (MRE). The article proposes new method of SM systems modelling and its application to the study of functioning of the production element (PE). The model allows to assess the reliability impact of PE on its performance under the following hypothesis: in case of PE failure the production service is interrupted, and after the performance restoration of the element the production service continues in consideration for the time of the interrupted service (non-devaluate failures) $[1,2]$.

The proposed method is based on the theorem presented below.

\section{Problem formulation}

Consider the SM system with the common phase space of states $M$. Select the two subsets $M_{+}$and $M_{-}$in the phase space of states $M$ of the SM processes, such that $M_{+} \cup M_{-}=M$. In the future, we will only talk about the subset $M_{+}$, as all the above will be true for the subset $M_{-}$. The times of single stay in the states $S_{i} \in M_{+}$are random variables (RV) $\alpha_{i}$ having mathematical expectations $m_{i}$, functions $F_{i}(t)$ and density $f_{i}(t)$ of distribution, with the images in the complex domain $F_{i}(s)$ and $f_{i}(s)$ respectively. RV $\theta_{\Sigma}-$ stay time period in the subset $M_{+}$having mathematical expectation $T_{+}$. The times of the multiple stays of the system in the state $S_{i} \in M_{+}$due to the repeated entering into them during the $\theta_{\Sigma}$ period are the $\mathrm{RV} \theta_{i}$ having mathematical expectations $m_{i}^{\theta}$, functions $F_{i}^{\theta}(t)$ and density $f_{i}^{\theta}(t)$ of distribution, with images in the complex domain $F_{i}^{\theta}(s)$ and $f_{i}^{\theta}(s)$ respectively. The distribution of embedded Markov chain (EMC) corresponds to the described SM process, and is characterized by specific frequency $\rho_{i}$ of entering each of the states $S_{i} \in M$ and $P_{i j}$ transition probabilities from states $S_{i} \in M$ to states $S_{j} \in M$. Not all the states $S_{i} \in M_{+}$can have the direct transition from $M_{+}$to $M_{-}$, that is why it is advisable to select the subset $E \subset M_{+}$of states $S_{e} \in E$ of the set $M_{+}$from which the direct transition into the set $M_{-}$is possible.

Let us introduce the definitions.

Trajectory - the set of states in which the system is to stay in order to go out of the subset $M_{+}$into $M_{-}$(or vice versa), and $M_{+} \cup M_{-}=M$ where $M$ - is the whole phase space of states. The trajectory of the system stay in the subset $M_{+}$starts in the state in which there is direct transition from the subset $M_{-}$. Such trajectory is ended up by the state, from which there is direct transition to the subset $M_{-}$. If such final state has two or more different transitions into various states of the subset $M_{-}$, than the two or more different trajectories are formed.

A particular trajectory is any part of the particular trajectory containing the state from which there is direct transition to the subset $M_{-}$will be referred to as particular trajectory of renewal.

The stationary distribution of EMC and transition probabilities are defined for each trajectory, assuming

\footnotetext{
* Corresponding author: zamoryonoff@gmail.com
} 
that the states of the trajectory form the set $M_{+}^{k} \subset M$, and all the other states are combined in the subset $M_{-}^{k}$ such that $M_{+}^{k} \cup M_{-}^{k}=M$, where $\mathrm{k}-$ is the number of trajectory. And also, the probabilities $P_{k}^{T}$ of the k-th trajectory on the basis of the transition probabilities of the system, and $\sum_{k} P_{k}^{T}=1$.

\section{Problem Solution}

The theorem on the times stay in the states of trajectory in view of the repeated returns.

If the discrete ergodic SM system with the known stationary distribution for the selected k-th trajectory with the states $S_{i} \in M_{+}^{k}$ the functions $F_{i}(t)$ and density $f_{i}(t)$ of distribution are known, the function $F_{i}^{k}(s)$ and the density $f_{i}^{k}(s)$ of the time distribution $\theta_{i}$ of the system stay in the state $S_{i}$ of this trajectory, taking into account repeated enterings it in the images domain are:

$$
\begin{aligned}
& F_{i}^{k}(s)=\frac{F_{i}(s)}{c_{i}^{k}-\left(c_{i}^{k}-1\right) f_{i}(s)}, \\
& f_{i}^{k}(s)=\frac{f_{i}(s)}{c_{i}^{k}-\left(c_{i}^{k}-1\right) f_{i}(s)}
\end{aligned}
$$

and in the original domain, they look like:

$$
\begin{aligned}
& F_{i}^{k}(t)=\frac{1}{c_{i}^{k}} F_{i}(t)+\frac{1}{c_{i}^{k}} \sum_{m=1}^{\infty}\left(1-\frac{1}{c_{i}^{k}}\right)^{m} F_{i}^{(*) m}(t), \\
& f_{i}^{k}(t)=\frac{1}{c_{i}^{k}} \cdot f_{i}(t)+\frac{1}{c_{i}^{k}} \sum_{m=1}^{\infty}\left(1-\frac{1}{c_{i}^{k}}\right)^{m} f_{i}^{(*) m+1}(t),
\end{aligned}
$$

where $c_{i}^{k}=\frac{\rho_{i}}{\rho_{e} P_{e j}}-$ is coefficient of increasing the stay time of the system in the states $S_{i} \in M_{+}$by re-entering it;

$\rho_{i}$ - is fixed rate of the system stay in the state $S_{i}$ for the selected trajectory;

$\rho_{e}$ - is fixed rate of the system stay in the state $S_{e}$ (a final state of the current trajectory from which there is direct transition to the subset $M_{-}$) for the selected trajectory;

$P_{e j}$ - is the probability of transition from state $S_{e}$ to $S_{j}$ belonging to the subset $M_{-}$.

Theorem proof.

Stay times of the system in the states $\left(S_{i} \in M_{+}^{k}\right)$ increase by the system re-entering with some probability in these states during the stay of the entire system in the subset $M_{+}^{k}$, and there is nothing else due to what they can increase which is obvious. But this means that the number of the system entering follows the geometrical law of distribution with the $P_{i}$ probability that the system will come out of this state, and the probability that it will remain is $1-P_{i}$. The graph that describes the behavior of the system in given state at certain distribution function $F_{i}(t)$ of the stay time in this state $S_{i}$ has the form shown in Fig. 1 .

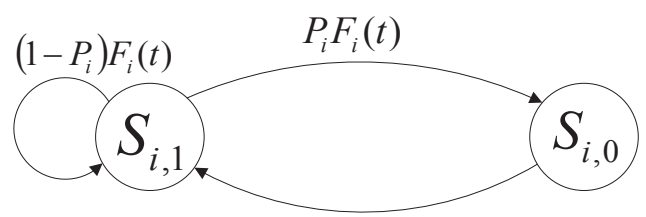

Fig. 1. Graph of states corresponding to the geometric distribution.

The legend are the following: $S_{i, 0}$ - the instantaneous state corresponds to the system coming out of state $S_{i}$; $S_{i, 1}$ - the studied state $S_{i}$, the total stay time in which it is being determined; $P_{i}$ - the probability of transition of the system from $S_{i, 1}$ to $S_{i, 0} ;\left(1-P_{i}\right)$ - the probability of the system re-entering the state $S_{i, 1} ; F_{i}(t)$ - is the DF of RV $\alpha_{i}$ having mathematical expectation $m_{i}$.

According to this graph let us make Markov renewal equation:

$$
F_{i}^{k}(t)=\left(1-P_{i}\right) \int_{0}^{t} f_{i}(t-s) F_{i}^{\theta}(s) d s+P_{i} F_{i}(t) .
$$

Iterating the resulting equation (3), we have [3]:

$$
F_{i}^{k}(t)=P_{i} F_{i}(t)+P_{i} \sum_{m=1}^{\infty}\left(1-P_{i}\right)^{m} f_{i}^{(*) m}(t) * F_{i}(t)
$$

The density of distribution $f_{i}^{k}(t)$ is as follows:

$$
f_{i}^{k}(t)=P_{i} \cdot f_{i}(t)+P_{i} \sum_{m=1}^{\infty}\left(1-P_{i}\right)^{m} f_{i}^{(*) m+1}(t)
$$

The unknown that is to be determined here is $P_{i}$.

It is known that the geometric distribution law leads to $m \theta_{i}=\frac{m_{i}}{P_{i}}$ and this leads to the following:

$$
P_{i}=\frac{m_{i}}{m \theta_{i}}
$$

Let us find $m \theta_{i}$.

According to the known theorem [1] $T_{+}$can be accurately determined. For the discrete states the formula is: 


$$
T_{+}=\frac{\sum_{i \in M_{+}} m_{i} \rho_{i}}{\sum_{e \in E \subset M_{+}} \sum_{j \in M_{-}} P_{e j} \rho_{i}}=\sum_{e \in M_{+}} m_{i} \frac{\rho_{i}}{\sum_{e \in E \subset M_{+}} \sum_{j \in M_{-}} P_{e j} \rho_{e}} .
$$

Then $m \theta_{i}^{k}$ :

$$
m \theta_{i}^{k}=m_{i} \cdot \frac{\rho_{i}}{\sum_{e \in E \subset M_{+}} \sum_{j \in M_{-}} P_{e j} \rho_{e}} .
$$

Let us introduce the coefficient $c_{i}^{k}$ of the increase of the system stay time in the states $S_{i}$ equal to

$$
c_{i}^{k}=\frac{\rho_{i}}{\sum_{e \in E \subset M_{+}} \sum_{j \in M_{-}} P_{e j} \rho_{e}} .
$$

Since there is only one way out for the current trajectory of the state $S_{e}$ - the final state of the trajectory from which there is direct transition to the state $S_{j}$ of the subset $M_{-}$, the coefficient $c_{i}^{k}$ of time increase spent by the system in the states $S_{i}$ would be:

$$
c_{i}^{k}=\frac{\rho_{i}}{\rho_{e} P_{e j}} .
$$

Then:

$$
m \theta_{i}^{k}=m_{i} \cdot c_{i}^{k},
$$

and the probability $P_{i}$ is determined by the condition of the increase $m_{i}$ up to the value $m \theta_{i}$ that is of equality $m \theta_{i}^{k}=m_{i} \cdot c_{i}^{k}$, which implies that the sought probability $P_{i}$ on the basis of (5) equals to:

$$
P_{i}=\frac{m_{i}}{m \theta_{i}^{k}}=\frac{1}{c_{i}^{k}} .
$$

Substituting (7) into (4) we obtain (2).

Applying the Laplace transformation to the formula (4), we obtain

$$
\begin{gathered}
F_{i}^{k}(s)=P_{i} F(s)+P_{i} \sum_{m=1}^{\infty}\left(1-P_{i}\right)^{m} f_{i}^{m}(s) F(s)= \\
=P_{i} F_{i}(s) \sum_{m=0}^{\infty}\left(1-P_{i}\right)^{m} f_{i}^{m}(s)
\end{gathered}
$$

The resulting expression is an infinitely decreasing geometric progression. Let us take the limit of the expression $\sum_{m=0}^{\infty}\left(1-P_{i}\right)^{m} f^{m}(s)$ :

$$
\lim _{n \rightarrow \infty} \sum_{m=0}^{n}\left(1-P_{i}\right)^{m} f_{i}^{m}(s)=\frac{1}{1-\left(1-P_{i}\right) f_{i}(s)} .
$$

Then the expression (8) will look like:

$$
F_{i}^{k}(s)=\frac{P_{i} F_{i}(s)}{1-\left(1-P_{i}\right) f_{i}(s)} .
$$

Accordingly, the image of DD $f_{i}^{k}(t)$ will look like:

$$
f_{i}^{k}(s)=\frac{s P_{i} F_{i}(s)}{1-\left(1-P_{i}\right) f_{i}(s)}=\frac{P_{i} f_{i}(s)}{1-\left(1-P_{i}\right) f_{i}(s)} .
$$

Substituting (7) into (9) and (10) we will obtain

$$
\begin{gathered}
F_{i}^{k}(s)=\frac{\frac{1}{c_{i}^{k}} F_{i}(s)}{1-\left(1-\frac{1}{c_{i}^{k}}\right) f_{i}(s)}=\frac{F_{i}(s)}{c_{i}^{k}-\left(c_{i}^{k}-1\right) f_{i}(s)}, \\
f_{i}^{k}(s)=\frac{\frac{1}{c_{i}^{k}} f_{i}(s)}{1-\left(1-\frac{1}{c_{i}^{k}}\right) f_{i}(s)}=\frac{f_{i}(s)}{c_{i}^{k}-\left(c_{i}^{k}-1\right) f_{i}(s)} .
\end{gathered}
$$

The theorem is proved.

Corollary 1.

The time $\theta_{\Sigma}^{T}$ of the system stay in the trajectory is the sum of all the RV $\theta_{i} \theta_{\Sigma}^{T}=\sum_{1}^{n} \theta_{i}$, which means that the DF $F_{\Sigma}^{T}$ of time $\theta_{\Sigma}^{T}$ - of the system stay in the trajectory is sequence of convolution of all DF $F_{i}^{\theta}$ :

$$
F_{\Sigma}^{T}=F_{0}^{\theta} * F_{1}^{\theta} * F_{2}^{\theta} * \ldots * F_{i}^{\theta} * \ldots * F_{n}^{\theta},
$$

where $\mathrm{n}-$ is the number of states included in the trajectory.

Corollary 2.

Based on the formula of total probability the expression for the distribution function $\theta_{\Sigma}$ of the system stay time in the subset $M_{+}$is:

$$
F_{\Sigma}=P_{1}^{T} \cdot F_{1}^{T}+P_{2}^{T} \cdot F_{2}^{T}+\ldots+P_{i}^{T} \cdot F_{i}^{T}+\ldots+P_{n}^{T} \cdot F_{n}^{T} .
$$

Corollary 3 .

The stay times in the states of the entire system are determined by the following expression:

$$
F_{i}^{\Sigma}=P_{1}^{T} \cdot F_{i}^{1}+P_{2}^{T} \cdot F_{i}^{2}+\ldots+P_{j}^{T} \cdot F_{i}^{j}+\ldots+P_{n}^{T} \cdot F_{i}^{n}
$$

Corollary 4.

DF of the stay times in the particular trajectories of renewal correspond to EMC for the distribution function of system stay times in the subset $M_{+}$with given initial state. Thus, the exact solution of the Markov renewal equation is obtained for the discrete SM system.

Note that in general, the algorithms of phase consolidation (for example, the one proposed in [2]) 
used for the transition from the systems with continuous set of states to the systems with discrete states are rough, that is why it is incorrect to speak about the exact solution of the Markov renewal equation in the continuous phase space.

The Method Of Trajectories

Step One. The transition from system with continuous states to system with discrete states $S_{i} \in M_{+}$. For that the DF $F_{i}$ of the system stay times in the new discrete states, $P_{i j}$ transition probabilities of these states to other states (transitional probabilities), the specific frequencies $\rho_{i}$ of entering the states (stationary distribution of EMC) and fixed probabilities of stay in the states (the stationary distribution of the SM process) are determined. The procedure is performed with the help of known methods of SM systems modelling.

Step Two. Selection of all possible trajectories of transition from the subset $M_{+}$to the subset $M_{-}$. Moreover, each state of the system is included in one or several trajectories at once.

Step Three. The stationary distribution of EMC and transition probabilities for each trajectory are determined.

Step Four. Based on the foregoing theorem, replaced by the stay times in the states $\alpha_{i}$ are replaced by $\theta_{i}$ - for them the densities and distribution function of system stay times in the states $S_{i} \in M_{+}$are determined, in view of the repeated returns in accordance with the presented theorem.

Step Five. In accordance with the theorem of total probability, the probabilities $P_{k}^{T}$ of each of the trajectories realization are determined on the basis of transition probabilities of the Markov chain.

Step Six. We find the distribution function of the stay time in $M_{+}$, regardless of the initial state, which is defined as the weighted sum (the mixture) of DF of each trajectory emerging from the state $S_{i}$. The coefficients of the mixture are found on the fifth step of the $P_{k}^{T}$ probability of trajectories.

Let us consider specific example implementation of the proposed method of modelling.

We will describe the operation of the element $[1,2]$. The service time per unit of output PE - RV $\alpha_{1}$ with the DF $F_{1}(t)=P\left\{\alpha_{1} \leq t\right\}$. Uptime PE - RV $\alpha_{2}$ with risk factors with DF $F_{2}(t)=P\left\{\alpha_{2} \leq t\right\}$, the renewal time of $\mathrm{PE}-\mathrm{RV} \beta_{2}$ with DF $G_{2}(t)=P\left\{\beta_{2} \leq t\right\}$. RV $\alpha_{1}, \alpha_{2}, \beta_{2}$ assumed to be independent having finite mathematical expectations and variances; DF $F_{1}(t), F_{2}(t), G_{2}(t)$ has densities $f_{1}(t), f_{2}(t), g_{2}(t)$. In case of PE failure the production service is interrupted, and after the performance restoration of the element the production service continues in consideration for the time of the interrupted service.

It is necessary to define the DF $F_{\varepsilon}(t) \mathrm{RV} \varepsilon$ - of the cycle time service of $\mathrm{PE}$ production unit in view of its failures, the mean and variance of said RV, as well as the performance of PE.

To describe the operation of the system we use the Markov renewal process (MRP) and the corresponding SM process (SMP) with the states:

$10 x-P E$ is operational, the service of the next production unit has begun; the remaining time before failure of PE, as well;

$11 \mathrm{x}$ - momentary state corresponding to the end of the maintenance unit of production; the remaining time before failure of PE, equals ;

20th - was restored and continued efficiency PE interrupted service production unit; the time remaining before the end of the interrupted service as well;

$21 \mathrm{x}$ - has failed PE, service unit of production is interrupted; the time remaining before the end of the interrupted service as well.

The transition graph of the system - in Fig. 2.

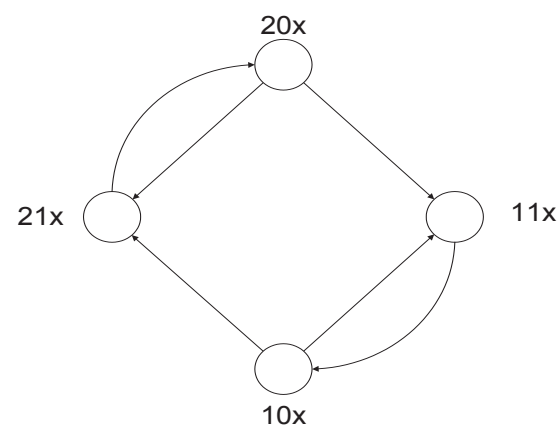

Fig. 2. The states graph of the system with continuous states.

The phase space of states is as follows:

$$
M=\{10 x, 11 x, 20 x, 21 x\} \text {. }
$$

We describe the densities and EMC transition probabilities:

$$
\begin{aligned}
& p_{10 x}^{11 y}=f_{1}(x-y), 0<y<x ; p_{10 x}^{21 y}=f_{1}(x+y), y>0 ; \\
& p_{20 x}^{21 y}=f_{2}(x-y), 0<y<x ; p_{20 x}^{11 y}=f_{2}(x+y), y>0 ; \\
& P_{11 x}^{10 x}=1 ; P_{21 x}^{20 x}=1 .
\end{aligned}
$$

We denote $\rho_{10}(x), \rho_{11}(x), \rho_{20}(x), \rho_{21}(x)$ of the density of the stationary distribution for the states of $10 \mathrm{x}$, $11 \mathrm{x}, 20 \mathrm{x}, 21 \mathrm{x}$, respectively. The system of integral equations for the densities of stationary distributions is:

$$
\left\{\begin{array}{c}
\rho_{11}(x)=\int_{x}^{\infty} f_{1}(y-x) \rho_{10}(y) d y+\int_{0}^{\infty} f_{2}(x+y) \rho_{20}(y) d y \\
\rho_{21}(x)=\int_{x}^{\infty} f_{2}(y-x) \rho_{20}(y) d y+\int_{0}^{\infty} f_{1}(x+y) \rho_{10}(y) d y \\
\rho_{10}(x)=\rho_{11}(x) ; \rho_{20}(x)=\rho_{21}(x) \\
\int_{0}^{\infty}\left(\rho_{10}(x)+\rho_{11}(x)+\rho_{20}(x)+\rho_{21}(x)\right) d x=1 .
\end{array}\right.
$$

In [1] it is shown that the solution of this system is determined by formulas 


$$
\rho_{10}(x)=\rho_{11}(x)=\rho_{0} \overline{F_{2}}(x) ; \rho_{20}(x)=\rho_{21}(x)=\rho_{0} \overline{F_{1}}(x),
$$

where the constant $\rho_{0}$ is explicitly from the normalization state.

Stay times in the states

$$
\begin{gathered}
v_{10 x}=x \wedge \alpha_{1} ; v_{11 x}=0 ; \\
v_{21 y}=\beta_{2} ; v_{20 y}=y \wedge \alpha_{2} .
\end{gathered}
$$

DF of the stay time in the states

$$
\begin{gathered}
\bar{F}_{10 x}(t)=\overline{1}_{x}(t) \cdot \bar{F}_{1}(t) ; F_{21 y}(t)=G_{2}(t) ; \\
\bar{F}_{20 y}(t)=\overline{1}_{y}(t) \cdot \bar{F}_{2}(t) .
\end{gathered}
$$

As mentioned above, let us complete the transition to an equivalent system with discrete states. The graph of such system is similar to the graph in Fig. 3. As shown in Fig. 3, the graph states have no continuous components, that is, the system is discrete. To go to discrete system phase consolidation algorithm (PCA) is used. It is proposed in [2].

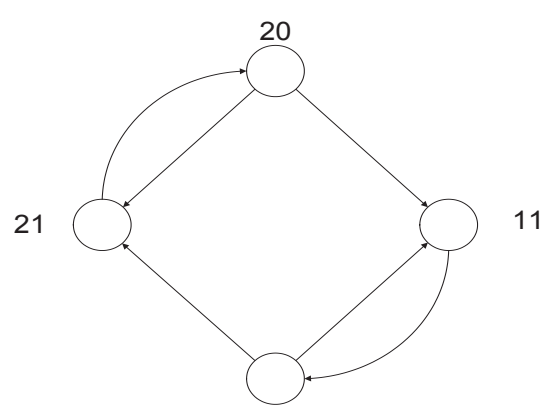

10

Fig. 3. The graph of the system states with discrete states.

It is necessary to define the transition probabilities, the stationary distribution of EMC and the stay times of distribution function in the discrete states 10 and 20 of the formulas [2]:

$$
\begin{gathered}
\hat{P}_{k, r}=\frac{\int_{E_{k}}^{\infty} \rho(d x) P\left(x, E_{r}\right)}{\rho\left(E_{k}\right)} \\
\hat{F}_{k}(t)=\frac{\int_{E_{k}} \rho(d x) F_{x}(t)}{\rho\left(E_{k}\right)}
\end{gathered}
$$

For the state 20:

$$
\rho_{21}=\rho_{20}=\rho_{0} \int_{0}^{\infty} \bar{F}_{1}(x) d x=\rho_{0} m_{\alpha_{1}}
$$

According to the formula (11) we find the transition probabilities:

$$
P_{20}^{21}=\frac{\int_{0}^{\infty} \bar{F}_{1}(x) F_{2}(x) d x}{m_{\alpha_{1}}} ; P_{20}^{11}=\frac{\int_{0}^{\infty} \bar{F}_{1}(x) \bar{F}_{2}(x) d x}{m_{\alpha_{1}}} .
$$

We find the DF system for stay time in the discrete state 20 using (12):

$$
\hat{F}_{20}(t)=\frac{\rho_{0} \int_{0}^{\infty} \bar{F}_{1}(x) d x\left[1-\overline{1}_{x}(t) \cdot \bar{F}_{2}(t)\right] d x}{\rho_{0} m_{\alpha_{1}}} .
$$

Let us transform the numerator:

$$
\int_{0}^{\infty} \bar{F}_{1}(x) d x\left[1-\overline{1}_{x}(t) \cdot \bar{F}_{2}(t)\right] d x=m_{\alpha_{1}} F_{2}(t)+\bar{F}_{2}(t) \int_{0}^{t} \bar{F}_{1}(x) d x .
$$

Here

$$
\hat{F}_{20}(t)=\frac{m_{\alpha_{1}} F_{2}(t)+\bar{F}_{2}(t) \int_{0}^{t} \bar{F}_{1}(x) d x}{m_{\alpha_{1}}} .
$$

For the state 10:

$$
\rho_{11}=\rho_{10}=\rho_{0} \int_{0}^{\infty} \bar{F}_{2}(x) d x=\rho_{0} m_{\alpha_{2}} .
$$

According to the formula (11) we find the transition probabilities:

$$
P_{20}^{11}==\frac{\int_{0}^{\infty} F_{1}(x) \bar{F}_{2}(x) d x}{m_{\alpha_{2}}} ; P_{20}^{21}==\frac{\int_{0}^{\infty} \bar{F}_{1}(x) \bar{F}_{2}(x) d x}{m_{\alpha_{2}}} .
$$

We find the DF system for stay time in the discrete state 10 using (12):

$$
\hat{F}_{10}(t)=\frac{\rho_{0} \int_{0}^{\infty} \bar{F}_{2}(x) d x\left[1-\overline{1}_{x}(t) \cdot \bar{F}_{1}(t)\right] d x}{\rho_{0} m_{\alpha_{2}}} .
$$

Let us transform the numerator:

$\int_{0}^{\infty} \bar{F}_{2}(x) d x\left[1-\overline{1}_{x}(t) \cdot \bar{F}_{1}(t)\right] d x=m_{\alpha_{2}} F_{1}(t)+\bar{F}_{1}(t) \int_{0}^{t} \bar{F}_{2}(x) d x$.

Here

$$
\hat{F}_{10}(t)=\frac{m_{\alpha_{2}} F_{1}(t)+\bar{F}_{1}(t) \int_{0}^{t} \bar{F}_{2}(x) d x}{m_{\alpha_{2}}}
$$

Let us define the stay time of distribution function of the system in the states 21 and 20 of the system based on the repeated enterings according to formula (1): 


$$
F_{20}^{2}(s)=\frac{\hat{F}_{20}(s)}{c_{20}-\left(c_{20}-1\right) \hat{f}_{20}(s)},
$$

where $c_{20}^{2}=\frac{\rho_{20}}{\rho_{20} \cdot P_{20}^{11}}=\frac{1}{P_{20}^{11}}$.

$$
F_{21}^{2}(s)=\frac{\hat{F}_{21}(s)}{c_{21}-\left(c_{21}-1\right) \hat{f}_{21}(s)},
$$

where $c_{21}^{2}=\frac{\rho_{21}}{\rho_{21} \cdot P_{20}^{11}}=\frac{1}{P_{20}^{11}}$.

There are two subsets:

$$
\begin{gathered}
M_{+}=\{10,21,20\} \\
M_{-}=\{11\}
\end{gathered}
$$

We define trajectory to the subset of system (Fig. 4):

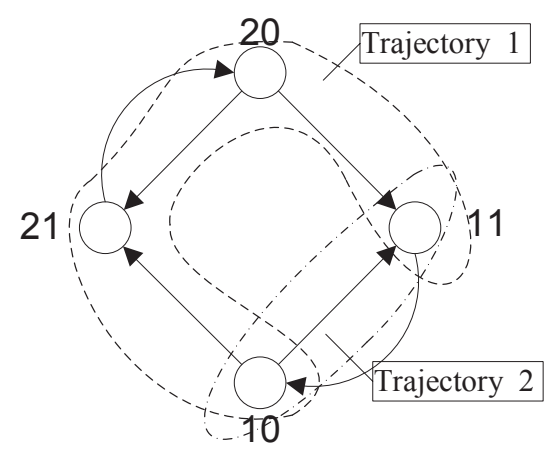

Fig.4. The trajectories of system subsets

$$
T_{1}=\left\{S_{10} S_{11}\right\}, \text { 4. } T_{2}=\left\{S_{10} S_{21} S_{10} S_{11}\right\} .
$$

With serial convolution we determine the stay time of distribution function of each trajectory.

$$
F_{1}^{T}(t)=F_{10}(t), F_{2}^{T}(t)=F_{10}(t) * F_{21}^{2}(t) * F_{20}^{2}(t),
$$

where * - sign of the convolution operation.

Let us find the probability of each of trajectories

$$
P_{1}^{T}=P_{10}^{11}, P_{2}^{T}=P_{10}^{21} .
$$

Hence, it becomes possible to determine the stay time of distribution function $F_{\Sigma}^{\theta}(t)$ of the system in the subset, regardless of the initial state as a weighted sum (a mixture) of the stay times of distribution function of the subset of each trajectory with coefficients equal to the probability of those trajectories:

$$
F_{\Sigma}^{\theta}(t)=F_{1}^{T}(t) \cdot P_{1}^{T}+F_{2}^{T}(t) \cdot P_{2}^{T} .
$$

Solutions for $F_{\text {обсл }}(t)$ DF cycle time maintenance PE because of its failure, and also for the mathematical expectation and variance obtained by known method using the Markov renewal equation, given in [4]:

$$
\begin{gathered}
F_{\text {обсл }}(t)=1-\frac{1}{M \alpha_{2}}\left[M \alpha_{2} \bar{F}_{1}(t)+\int_{0}^{t} \overline{F_{2}}(x) d x \int_{x}^{t} f_{1}(s) \overline{G_{2}}(t-s) d s+\right. \\
\left.+\sum_{n=1}^{\infty} \int_{0}^{t} \overline{F_{2}}(x) d x \int_{x}^{t} f_{1}(s) F_{2}^{\bullet(n)}(s-x) d s \int_{s}^{t} g_{2}^{\bullet(n)}(y-s) \overline{G_{2}}(t-y) d y\right] . \\
M \theta=\frac{M \alpha_{1}\left(M \alpha_{2}+M \beta_{2}\right)}{M \alpha_{2}} \\
D \theta=\left(1+\frac{2 M \beta_{2}}{M \alpha_{2}}\right) D \alpha_{1}+\frac{M \alpha_{1}}{M \alpha_{2}}\left(D \beta_{2}+\right. \\
\left.+\left(M \beta_{2}\right)^{2}\left(1-\frac{M \alpha_{1}}{M \alpha_{2}}\right)\right)+\frac{2\left(M \beta_{2}\right)^{2}}{M \alpha_{1}} \int_{0}^{\infty} \bar{F}_{1}(s) H_{2}(s) d s .
\end{gathered}
$$

Results comparing the two methods are presented in Fig. 5.

The initial data for the modelling are:

DF, and distributed by the generalized Erlang law of second order with the parameters $v_{1}, v_{2} ; \lambda_{1}, \lambda_{2} ; \mu_{1}, \mu_{2}$ respectively. Moreover,

$$
f_{1}(t)=\frac{v_{1} v_{2}\left(e^{-v_{1} t}-e^{-v_{2} t}\right)}{v_{2}-v_{1}},
$$

where $v 1=5,505(\mathrm{~h}-1) ; \mathrm{v} 2=54,495(\mathrm{~h}-1)$.

$$
f_{2}(t)=\frac{\lambda_{1} \lambda_{2}\left(e^{-\lambda_{1} t}-e^{-\lambda_{2} t}\right)}{\lambda_{2}-\lambda_{1}},
$$

where $\lambda 1=0,0275(\mathrm{~h}-1) ; \lambda 2=0,272(\mathrm{~h}-1)$;

$$
g_{2}(t)=\frac{\mu_{1} \mu_{2}\left(e^{-\mu_{1} t}-e^{-\mu_{2} t}\right)}{\mu_{2}-\mu_{1}},
$$

where $\mu 1=0,667$ (h-1); $\mu 2=2,000$ (h-1).

Fig. 5 shows the modelling results.

Fig. 5a shows that the results of the proposed modelling of the exact method and of the approximate one almost coincide. It is possible to see the difference in the curves only with 4,000-times magnification in Fig. $5 b$.

Mathematical expectation and variance RV -time cycle service of PE production unit in view of its failures is defined in two ways:

- known method using the Markov renewal equation:

$m_{\text {обслл }}=0.209999999 \mathrm{~h} ; D_{\text {обслл }}=0.069076$;

- the proposed method:

$m_{\Sigma}^{\theta}=0.210000000 h ; D_{\Sigma}^{\theta}=0.069423$. 


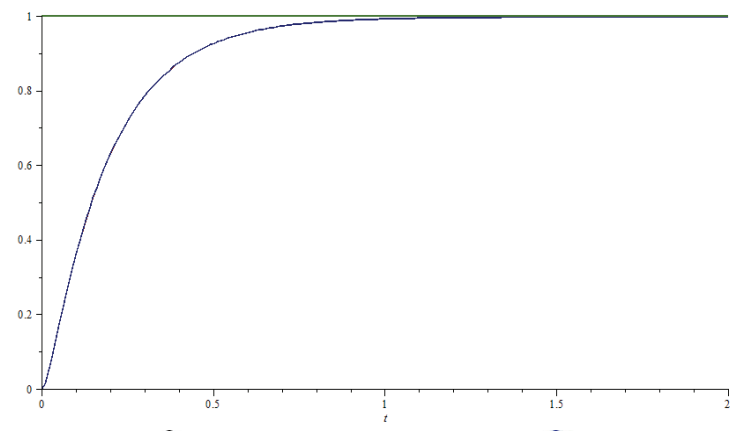

a)

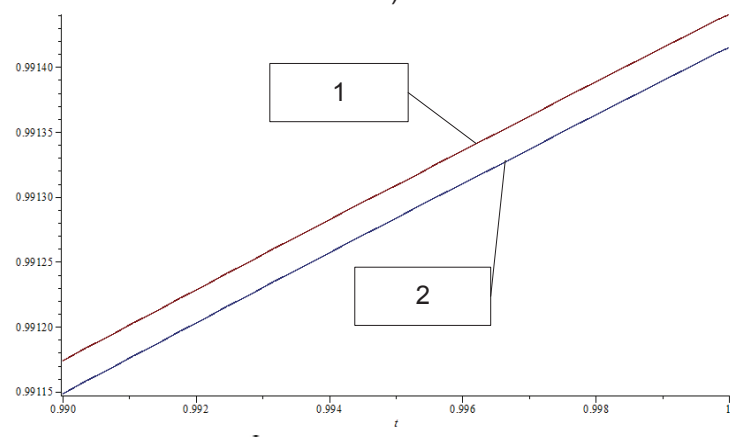

b)

Fig. 5. Comparison of the modelling results with the help of the classic approximate method of successive approximations, and of the precise method proposed: 1 - with approximate method; 2 - the solution offered with the precise method.

\section{Conclusions}

The results of modelling prove the correctness of the proposed method. The obtained exact solution is of great interest for it allows to determine accurately the DF of time between the events in the stream of the resulting superposition of the renewal stream and alternating renewal stream. In the future it is expected to approve the proposed method for the modelling of other systems.

The research was partially supported by the Russian Foundation for Basic Research (research project No. 15-0105840 a.).

\section{References}

1. V.S. Korolyuk, A.F. Turbin, Markov renewal processes in reliability problems of systems, Naukova Dumka (1982)

2. V.S. Korolyuk, A.F. Turbin, Stochastic models of systems, Naukova Dumka (1989)

3. V.Y. Kopp, M.V. Zamoryonov, U.E. Obzherin, O.V. Filipovich, Methods for semi-Markov systems modelling, Adaptive Systems of automatic control: Interdepartmental sci-tech collection. №1 (26). pp. 208-221 (2015)

4. V.Y. Kopp, Y.E Obzherin, A.I. Peschanskiy, Automated lines modelling (Sevastopol University SevNTU, 2006) 